\title{
An Iterative Method for Solving Quadratic Optimal Control Problem Using Scaling Boubaker Polynomials
}

\author{
Imad Noah Ahmed ${ }^{1 *}$, Eman Hassan Ouda ${ }^{1}$ \\ ${ }^{1}$ University of technology, Baghdad, Iraq \\ *Corresponding author: Imad Noah Ahmed: imadahmed86@yahoo.com
}

\section{OPEN ACCESS}

Citation: Ahmed I.N., Ouda E.H (2020) An Iterative Method for Solving Quadratic Optimal Control Problem Using Scaling Boubaker Polynomials. Open Science Journal 5(2)

Received: $9^{\text {th }}$ June 2020

Accepted: $18^{\text {th }}$ June 2020

Published: $30^{\text {th }}$ June 2020

Copyright: (c) 2020 This is an open access article under the terms of the Creative Commons Attribution License, which permits unrestricted use, distribution, and reproduction in any medium, provided the original author and source are credited.

Funding: The author(s) received no specific funding for this work

Competing Interests: The author has declared that no competing interests exists.
Abstract:

In this paper, an iterative method was used for solving a quadratic optimal control problem (QOCP) by the aid of state parameterization technique and scaling Boubaker polynomials. Some numerical examples were added to show the applicability of the method, also a comparison with other method was presented. The process steps were illustrated by some numerical examples with graphs done by Matlab.

Keywords: Quadratic optimal control problem, State parameterization technique, Scaling Boubaker function, Iterative method.

\section{Introduction}

Optimal control represents a large field in which many researchers gave different methods of various aspects. The crucial aim for solving an optimal control problem (OCP) is to find the control variable which minimizes a given performance index while all the given constraints are satisfied. State parameterization method is one of the mostly used direct methods in solving (OCPs) by the researchers. Kafash B., Delavarkhalafi A. and Karbassi S. M., used state parameterization for solving (NOCPs) and the controlled Duffing Oscillator [1]. Ouda E. H., utilized generalized Laguerre polynomials as a basis function with the aid of state-control parameterization to find an approximate solution for (OCPs) [2].

Scaling functions are a functional dilation equation has the general following form

$$
f(t)=\sum_{k=0}^{N} C_{k} f(2 t-K), \quad 0 \leq t \leq 1, C_{k} \text { are real or complex coefficients ... (1) }
$$


With a nonzero solution, this kind of equations has been used in many fields (e.g., interpolating subdivision schemes and wavelet theory) [3]. Yousfi S. A., presented a numerical solution of Emden-Fowler equations using Legendre scaling function approximation [4]. Ouda E. and Ahmed I. utilized direct methods (state and control Parameterization) with the scaling Boubaker function for solving OCP[5].

Iterative technique was also largely used for solving OCPs in last decades, Keyanpour M. and Azizsefat M., had an iterative approach with a hybrid of perturbation and parameterized methods for this purpose [6]. Ramezanpour H. et al., presented a new procedure depending on homotopy perturbation method with iterative technique to solve an optimal control problem of a bilinear systems [7]. Elaydi H., Jaddu H. and Wadi M., utilized Legendre scaling function with iterative technique for solving NOCP [8]. Jaddu H. and Majdalawi A., presented an iterative technique in two proceedings, firstly by parameterizing the state variables by finite length Chebyshev series [9], secondly by a finite length Legendre polynomials [10].

Eskandri M. et al., introduced a method for solving a class of nonlinear quadratic optimal control problem (NQOCP) based on variational iteration method [11]. Ramezani M., proposed a new iterative method utilizing 2nd kind Chebyshev wavelet [12]. Shihab S. and Delphi M., used the iterative technique on B-spline Bernestein polynomials [13].

Boubaker polynomials are proved to be a good tool for solving (OCPs), Samaa F. et al., used indirect method based on Boubaker polynomial [14]. Ouda E. H., deduced the operational matrices of derivative and integration and using them with the indirect method [15]. Many other researchers deal with this kind of polynomials in different proceedings. The novelty of our approach is using scaling Boubaker polynomials for solving ( $\mathrm{QOCP}$ 's), this method was proved to be efficient and accurate. A comparison was introduced to show the capability of this method with some other methods.

This paper is arranged as follows, in section2, Boubaker polynomials have been presented. In section3, scaling Boubaker polynomials were introduced. In section 4, the proposed method was presented in steps. In section 5, some numerical examples with comparison for the first example and illustrative figures were added to show the capability of this method, at the end conclusion and the references.

\section{Boubaker polynomials}

The Boubaker polynomials were established for the first by Boubaker et al. as a tool for solving heat equation inside physical model, and then it was used for solving different equations in many applications. [16]

Boubaker polynomial is introduced by the following equation [17]

$B_{n}(t)=\sum_{p=0}^{\zeta(n)}\left[\frac{(n-4 p)}{(n-p)} C_{n-p}^{p}\right]{ }_{(-1)}^{p} t^{n-2 p}, n=0,1,2, \ldots$

where $\quad C_{n-p}^{p}=\frac{(n-p) !}{p !(n-2 p) !}, \quad \zeta(n)=\left\lfloor\frac{n}{2}\right\rfloor=\frac{2 n+\left((-1)^{n}-1\right)}{4}$.

$m$ is the degree of Boubaker polynomials . 
The first terms of Boubaker polynomials are

$$
B_{0}(t)=1, \quad B_{1}(t)=t, \quad B_{2}(t)=t^{2}+2, \ldots
$$

and the recurrence relation $B_{m}(t)=t B_{m-1}(t)-B_{m-2}(t)$, where $m>2$

\section{Scaling boubaker polynomials (SBP)}

The Scaling Boubaker polynomials (SBP), can be defined as follows [18]

$$
S B_{n m}(t)=\left\{\begin{array}{cl}
2^{\frac{k}{2}} B_{m}\left(2^{k+1} t-2 n-1\right) & \frac{2 n-1}{2^{k+1}} \leq t \leq \frac{2 n}{2^{k+1}} \\
0 & \text { Otherwise }
\end{array} .\right.
$$

The arguments of scaling $(k, n, m, t), \mathrm{k}$ is positive integer, $\mathrm{n}=0,1,2,3, \ldots$, $2 \mathrm{k}, \mathrm{m}$ is degree of Boubaker polynomials and $\mathrm{t}$ is the time.

Choosing $k=1$ and $m=5$. The first five terms Scaling Boubaker $\mathrm{SB}_{\mathrm{m}}(\mathrm{t})$ were found by using (3) to be:

$$
\begin{aligned}
& S B_{0}=\sqrt{2}, \\
& S B_{1}=\sqrt{2}(4 t-1) \\
& S B_{2}=\sqrt{2}\left(16 t^{2}-8 t+3\right), \\
& S B_{3}=\sqrt{2}\left(64 t^{3}-48 t^{2}+16 t-2\right), \\
& S B_{4}=\sqrt{2}\left(256 t^{4}-256 t^{3}+96 t^{2}-16 t-1\right) .
\end{aligned}
$$

The convergence of this method with state parameterization technique has been treated in [2].

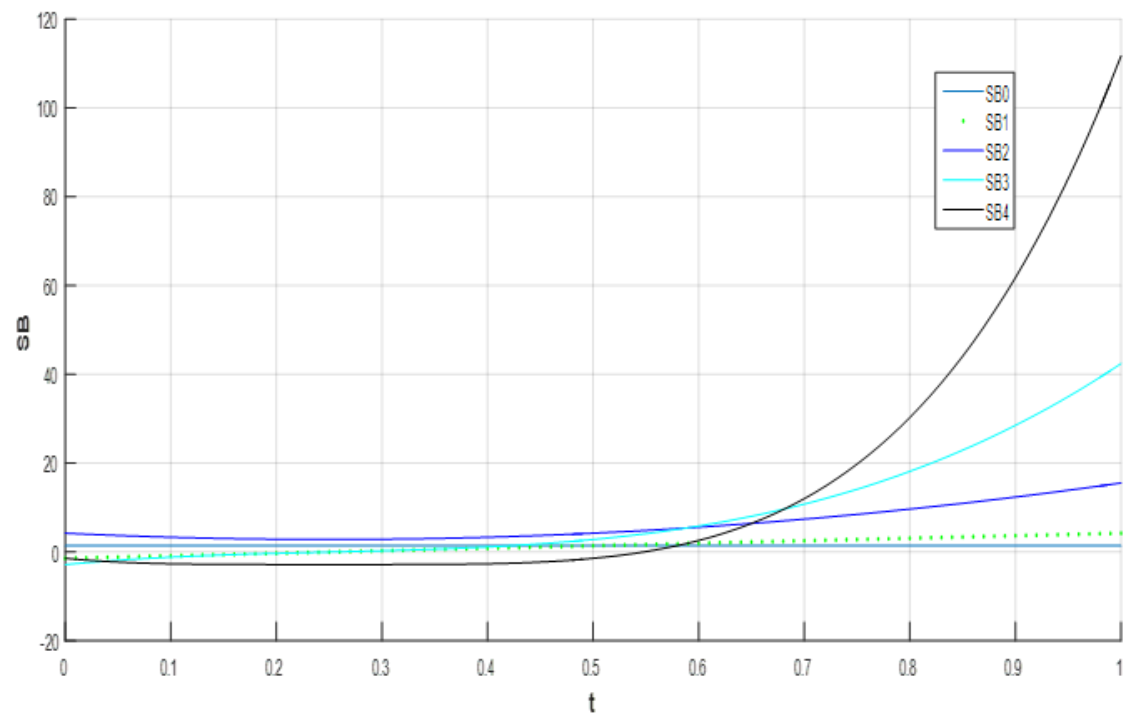

Figure 1.Scaling Boubaker polynomials $(\mathrm{n}=4)$ 


\section{The proposed method}

The state parameterization is based on approximating state variables by using Scaling Boubaker polynomials (SBP) with unknown coefficients as follows,

$$
x(t)=\sum_{i=0}^{m} a_{i} S B_{i}(t)=a^{T} S B(t) \quad t_{0} \leq t \leq t_{f}
$$

where $a_{i} s$ are unknown coefficients of state, SB are the Scaling Boubaker polynomials.

$$
x(t)=a_{0} S B_{0}+a_{1} S B_{1}+a_{2} S B_{2}+a_{3} S B_{3}+\ldots+a_{n} S B_{n}
$$

with initial condition $x\left(t_{0}\right)=\sum_{i=0}^{4} a_{i} S B_{i}\left(t_{0}\right)=x_{0}$, where the state coefficients $a_{i} s$ must be found.

The process steps are

- For the 1st iteration, representing the unknown function $\mathrm{x}$ by the first three terms of the scaling Boubaker polynomial with their coefficients $a_{i}{ }^{\prime} s$ on the interval $[0,1]$ as

$$
x(t)=a_{0} S B_{0}+a_{1} S B_{1}+a_{2} S B_{2}
$$

- $\quad$ Substituting (5) in constraint equation for finding the control variable $\mathrm{u}$ with the unknown coefficients $a_{i}{ }^{\prime} s$.

- Using now the performance index to find J, we get a set of algebraic equations with the $a_{i}{ }^{\prime} s$. which can simply be found by the aid of the initial conditions.

- Repeating the first iteration with more terms of the scaling Boubaker polynomial for the second and third iterations, evaluating the $J_{i}$ 's and compare their values with respect to $J$ exact.

- The iterative process continues until a predetermined acceptable error value.

\section{Numerical Examples}

Ex.1: Consider the following quadratic optimal control problem [ 19 ]

Min $I=\int_{0}^{1}\left(x^{2}+u^{2}\right) d t$

with $u=\dot{x} \quad$ and $\quad x(0)=0, \quad x(1)=\frac{1}{2}$.

The exact solution is

$x_{\text {exact }}(t)=\frac{e\left(e^{t}-e^{-t}\right)}{2\left(e^{2}-1\right)}, \quad u_{\text {exact }}(t)=\frac{e\left(e^{t}+e^{-t}\right)}{2\left(e^{2}-1\right)}, J_{\text {exact }}=0.328258821374830$

The results of the $1^{\text {st }}$ Iteration are

$x_{1}=(5 / 44) t^{2}+(17 / 44) t+(1 / 1306897830861018)$

$u_{1}=(5 / 22) t+(17 / 44)$

$J_{1}=0.328598484848489$ 
The results of the $2^{\text {nd }}$ Iteration are

$x_{2}=(7 / 86) t^{3}-(4 / 473) t^{2}+(202 / 473) t+(1 / 317825433919741)$

$u_{2}=(21 / 86) t^{2}-(8 / 473) t+(202 / 473)$

$J_{2}=0.328259337561646$

The results of the $3^{\text {rd }}$ Iteration are $x_{3}=(21 / 2242) t^{4}+(145 / 2314) t^{3}+(115 / 41138) t^{2}+(483 / 1136) t+(1 / 704329587969285)$ $u_{3}=(42 / 1121) t^{3}+(435 / 2314) t^{2}+(115 / 20569) t+(483 / 1136)$ $J_{3}=0.328258830708990$

From table1, we noticed that our proposed method has less absolute error with respect to Delphi and Mehne in which power and Bernestein polynomials have been used.

Table 1.The values of cost functional $\mathrm{J}$ in Example 1

\begin{tabular}{||c||c||c|c||c||}
\hline \hline Iteration & $\begin{array}{c}\text { J(approximate) } \\
\text { proposed method }\end{array}$ & $\begin{array}{c}\text { Absolute error } \\
\text { for our method }\end{array}$ & Delphi Method [19] & $\begin{array}{c}\text { Mehne } \\
\text { Method } \\
{[20]}\end{array}$ \\
\hline \hline 1 & 0.328598484848489 & $3.39663 \times 10^{-4}$ & $3.379 \times 10^{-4}$ & $\begin{array}{c}5 \\
.0 \times 10^{-3}\end{array}$ \\
\hline 2 & 0.328259337561646 & $5.16186 \times 10^{-7}$ & $2.1814 \times 10^{-4}$ & $3.4 \times 10^{-3}$ \\
\hline 3 & 0.328258830708990 & $9.33416 \times 10^{-9}$ & $2.03089 \times 10^{-4}$ & $2.1 \times 10^{-4}$ \\
\hline
\end{tabular}

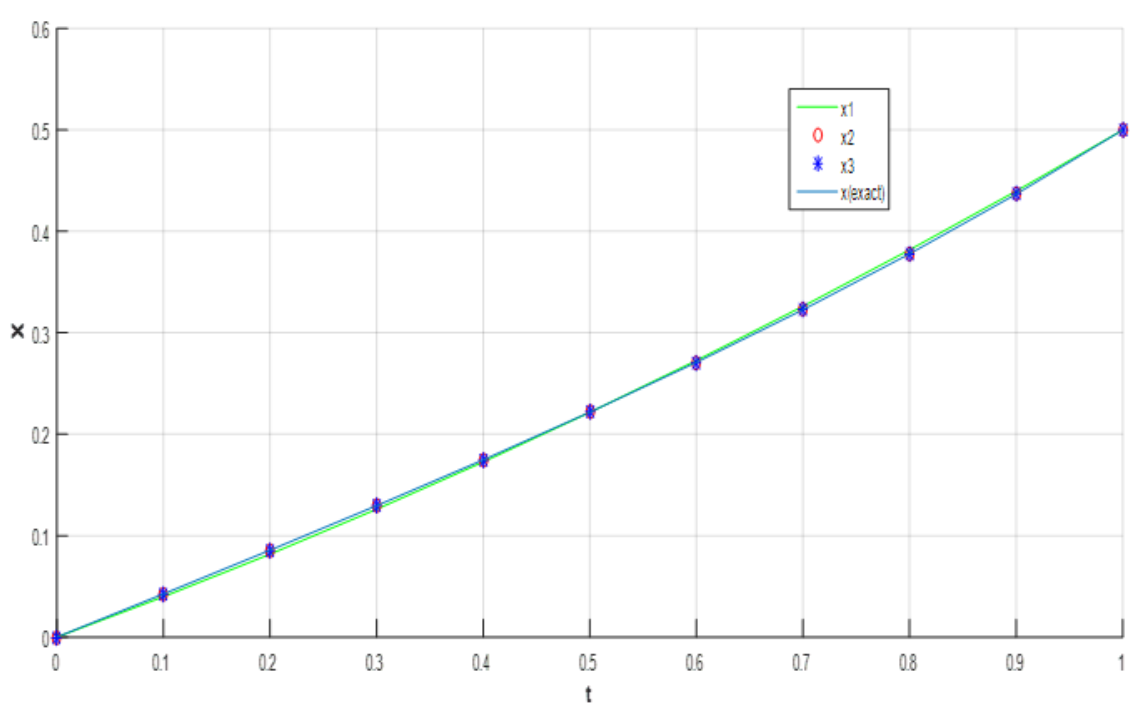

Figure 2 Example 1 - x variables 


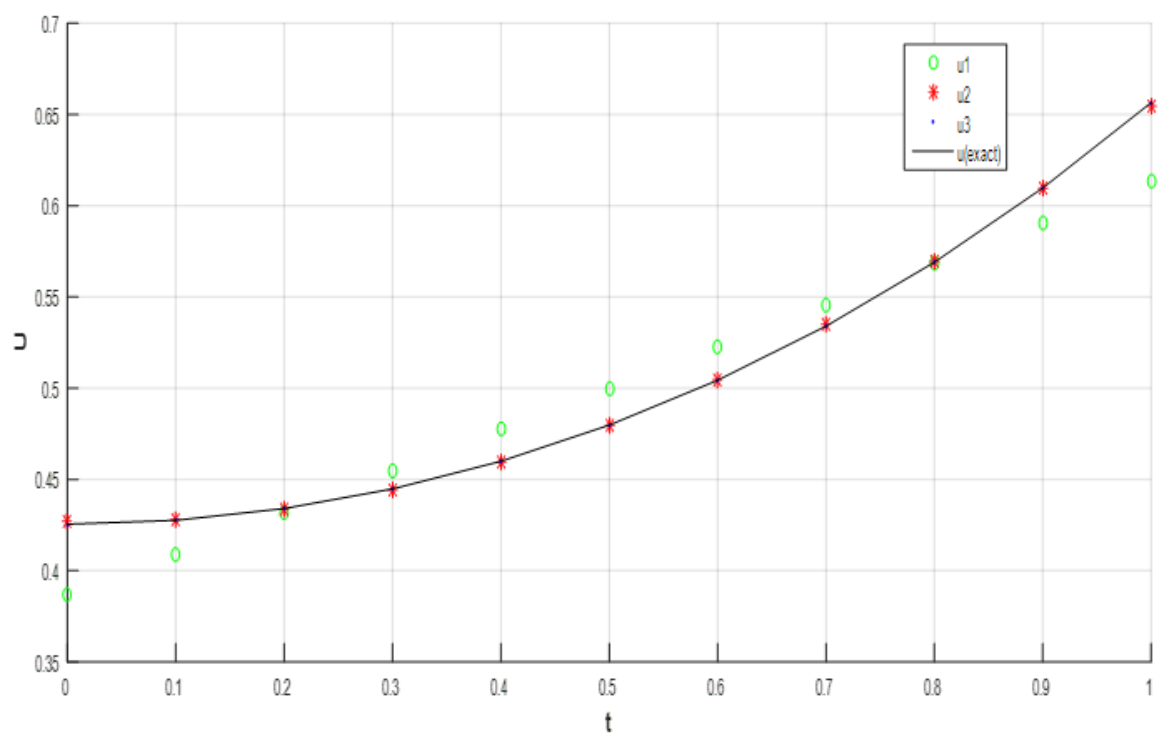

Figure 3 Example $1-\mathrm{u}$ variables

Ex.2: Consider the problem [21]

$\operatorname{Min} l=\frac{1}{2} \int_{0}^{1}\left(x^{2}+u^{2}\right) d t \quad, 0 \leq t \leq 1$.

$u=\dot{x}+x$ with $x(0)=1$

The exact solution is $x_{\text {emment }}(t)=A e^{\sqrt{2} t}+(1-A) e^{-\sqrt{2} t}$

$u_{\text {mannt }}(t)=A(\sqrt{2}+1) e^{\sqrt{2} t}-(1-A)(\sqrt{2}-1) e^{-\sqrt{2} t}$, where

$A=\frac{2 \sqrt{2}-3}{2 \sqrt{2}-\left(e^{\sqrt{2})^{2}}-3\right.}$

$J_{\text {exact }}=0.192909298093169$

The results of the $1^{\text {st }}$ Iteration are

$x_{1}=(100 / 187) t^{2}-(234 / 187) t+1$

$u_{1}=(100 / 187) t^{2}-(2 / 11) t-(47 / 187)$

$J_{1}=0.194295900178253$

The results of the $2^{\text {nd }}$ Iteration are $x_{2}=(634 / 2775) t^{3}+(1125 / 1283) t^{2}-(757 / 554) t+1$

$u_{2}=-(634 / 2775) t^{3}+(1965 / 10264) t^{2}+(907 / 2342) t-203 / 554$

$J_{2}=0.192931605611848$

The results of the $3^{\text {rd }}$ Iteration are $x_{3}=(113 / 1297) t^{4}-(772 / 1917) t^{3}+(1014 / 1033) t^{2}-(1128 / 815) t+1$

$u_{3}=(113 / 1297) t^{4}-(335 / 6179) t^{3}-(111 / 490) t^{2}+(1262 / 2179) t-(313 / 815)$

$J_{3}=0.192909445024077$

Table 2 Numerical Results of Example 2

\begin{tabular}{|c||c||c||}
\hline Iteration & $\mathrm{J}$ (approximate) & Absolute error \\
\hline \hline 1 & 0.194295900178253 & $1.3866 \times 10^{-3}$ \\
\hline 2 & 0.192931605611848 & $2.23075 \times 10^{-5}$ \\
\hline 3 & 0.192909445024077 & $1.46930 \times 10^{-7}$ \\
\hline
\end{tabular}




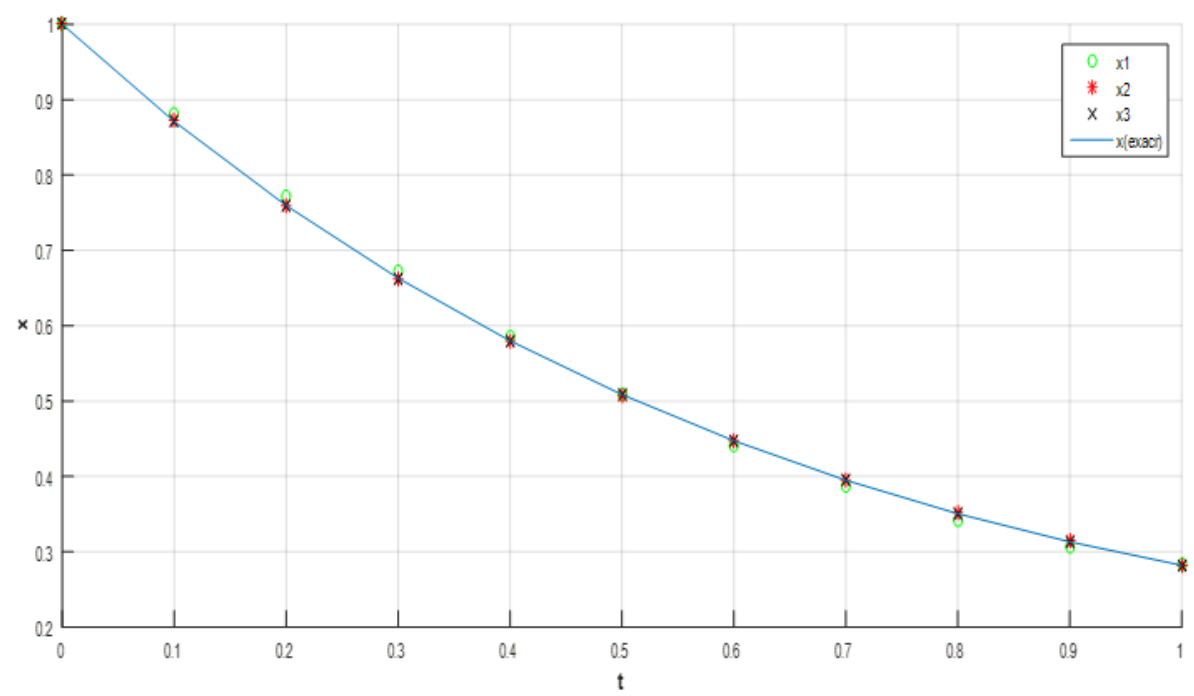

Figure 4 Example 2-x variables

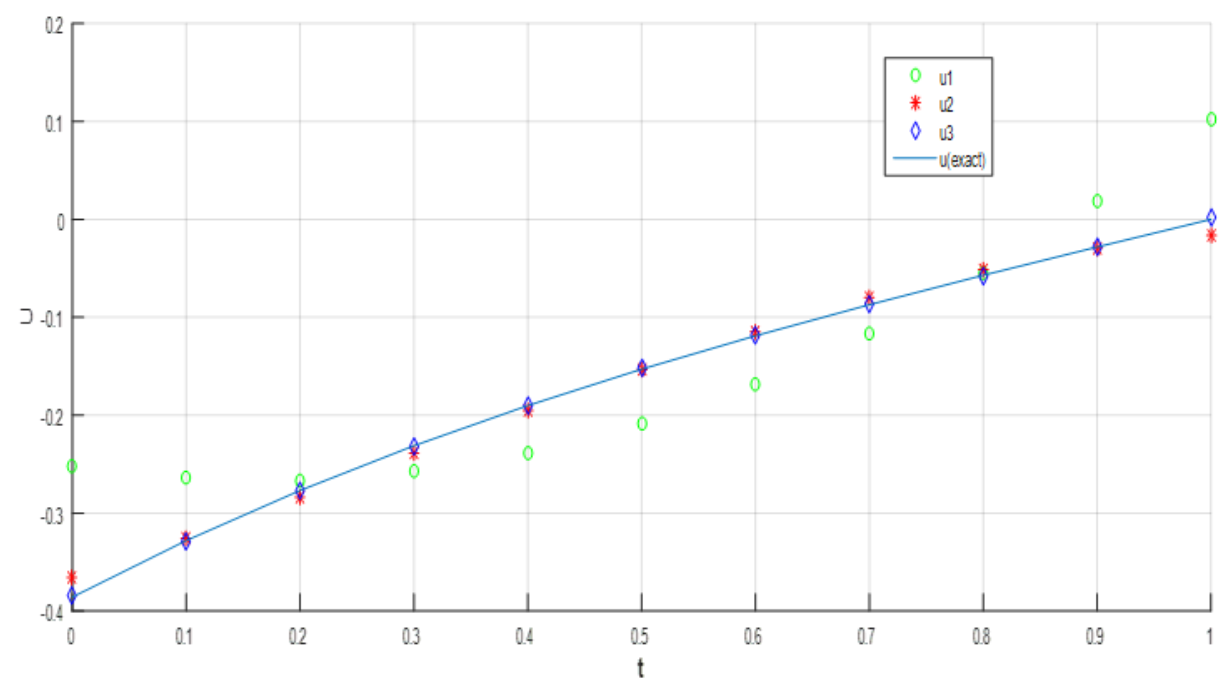

Figure 5 Example 2- u variables

Ex.3: Consider the problem [2]

$$
\begin{aligned}
& \text { Min } I=\frac{1}{2} \int_{0}^{1}\left(2 x^{2}+u^{2}\right) d t, 0 \leq t \leq 1 . \\
& u=\dot{x}-\frac{x}{2}, \quad x(0)=1,
\end{aligned}
$$

and

$$
x_{\text {exact }}=\frac{2 e^{\mathrm{st}}+e^{\mathrm{s}}}{e^{\mathrm{st}}\left(2+e^{\mathrm{s}}\right)}, u_{\text {exact }}=\frac{2\left(e^{\mathrm{st}}-e^{\mathrm{s}}\right)}{e^{\frac{\mathrm{st}}{\mathrm{a}}\left(2+e^{\mathrm{s}}\right)}}, \quad J_{\text {exact }}=0.864164497
$$

The results of the $1^{\text {st }}$ Iteration are

$x_{1}=(54 / 73) t^{2}-(412 / 365) t+1$

$u_{1}=(746 / 365) t-(27 / 73) t^{2}-(1189 / 730)$

$J_{1}=0.864726027397260$ 
The results of the $2^{\text {nd }}$ Iteration are

$$
\begin{aligned}
& x_{2}=-(233 / 1673) t^{3}+(1067 / 1126) t^{2}-(625 / 521) t+1 \\
& u_{2}=(233 / 3346) t^{3}-(691 / 775) t^{2}+(2001 / 802) t-(1771 / 1042) \\
& J_{2}=0.864218070459266
\end{aligned}
$$

The results of the $3^{\text {rd }}$ Iteration are

$$
\begin{aligned}
& x_{3}=(295 / 2182) t^{4}-(841 / 2053) t^{3}+(1471 / 1325) t^{2}-(919 / 749) t+1 \\
& u_{3}=-(266 / 3935) t^{4}+(1741 / 2335) t^{3}-(1586 / 889) t^{2}+(2678 / 945) t-(1031 / 597) \\
& J_{3}=0.864164568963970
\end{aligned}
$$

Table 3 Numerical Results of Example 3

\begin{tabular}{||c||c|c|}
\hline \hline Iteration & J (approximate) & Absolute error \\
\hline \hline 1 & 0.864726027397260 & $5.61530 \mathrm{e} \times 10^{-4}$ \\
\hline 2 & 0.864218070459266 & $5.35734 \mathrm{e} \times 10^{-5}$ \\
\hline 3 & 0.864164568963970 & $7.19639 \mathrm{e} \times 10^{-8}$ \\
\hline
\end{tabular}

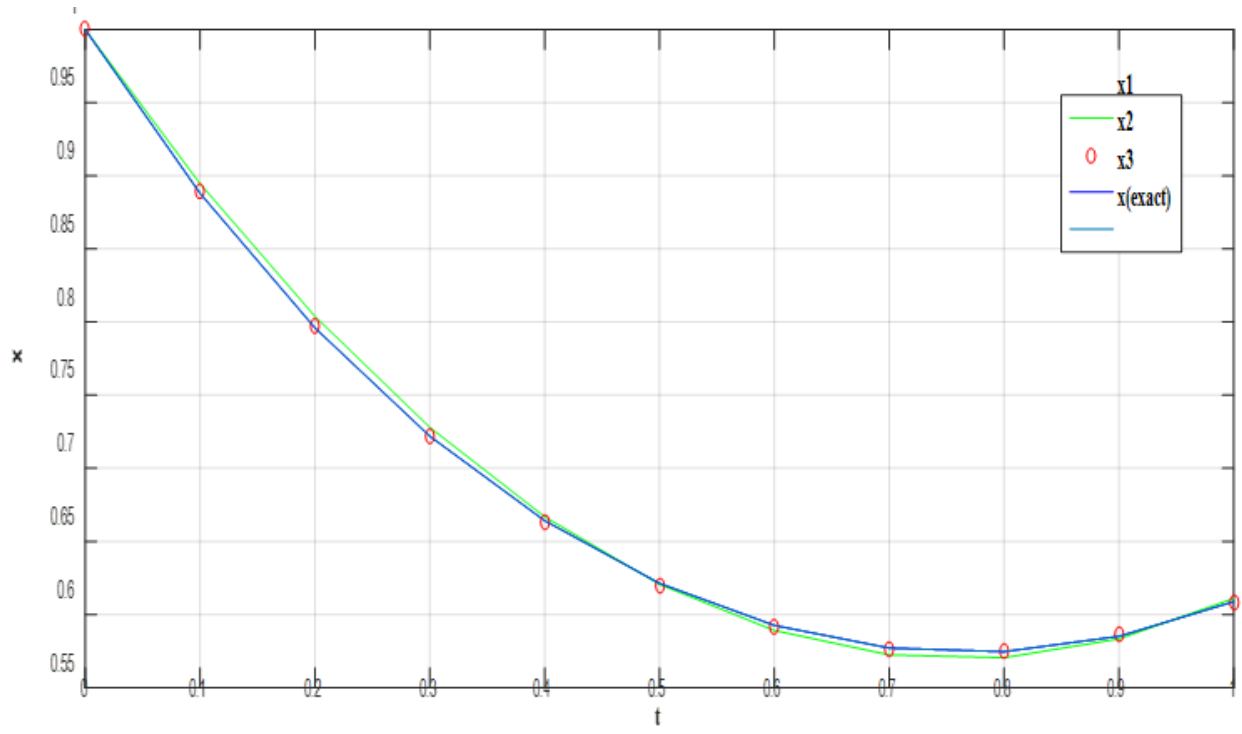

Figure 6 Example3 -x variables 


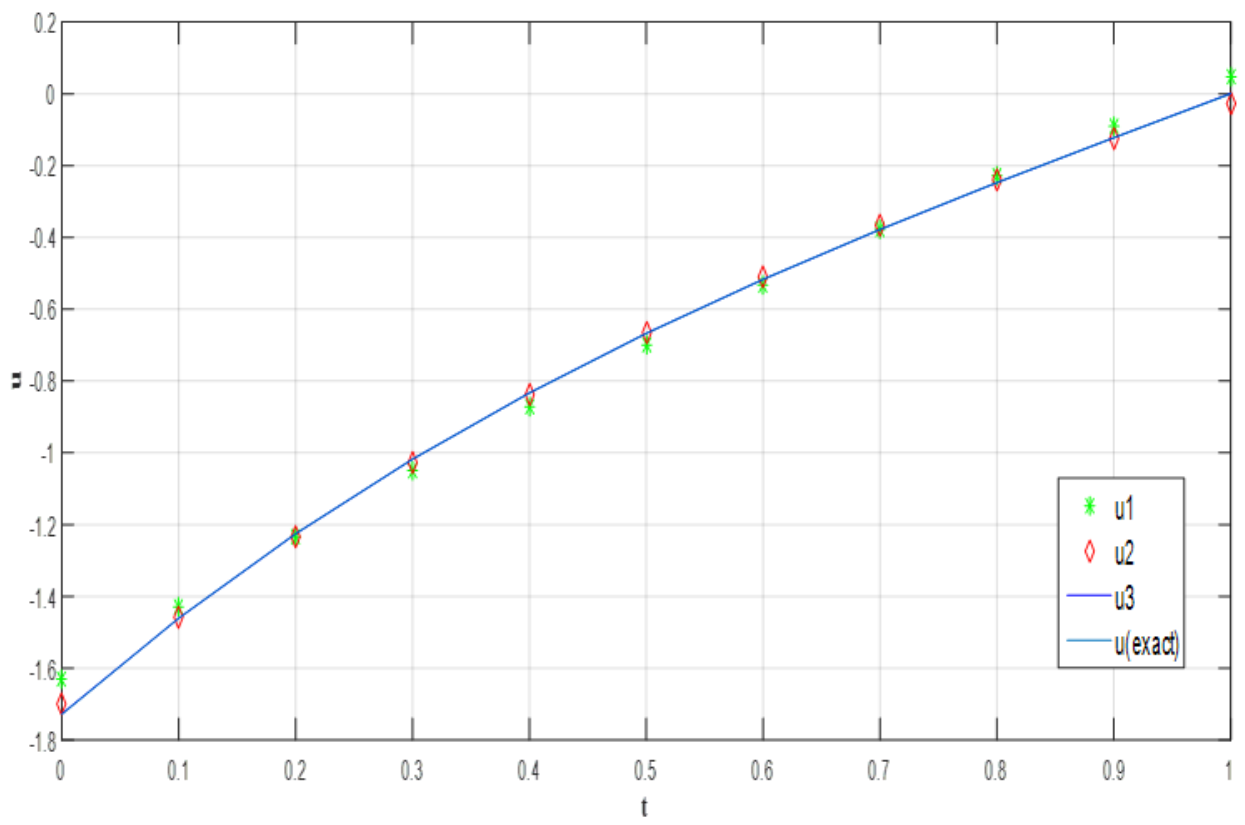

Figure 7 Example 3 - u variables

\section{Conclusion}

An iterative method with state parameterization technique using scaling Boubaker polynomials was proved to be a good tool for evaluating the optimal solution of (QOCP) by its rapid convergence and simplicity. The numerical examples show the applicability and accuracy of this method, also comparison with other methods proves its efficiency.

\section{References:}

1.Kafash B., Delavarkhalafi A. and Karbassi S. M., Numerical Solution of Nonlinear Optimal Control Problems Based on State Parameterization, Iranian Journal of Science and Technology, Vol.A3,pp.331-340(2012).

2.Ouda E. H., The Efficient Generalized Laguerre Parameterization for Quadratic Optimal Control Problem, Journal of College of Education, Al-Mustansyriah University, vol.3, Issue (1812-0380), PP.263-276(2014).

3.Colella D. and Heil Ch., Characterizations of Scaling Functions: Continuous Solutions, SIAM Jour. of Matrix Anal. Appl., Vol. 15, No. 2, pp. 496-518, (1994).

4.Yousefi S. A., Legendre Scaling Function for Solving Generalized Emden-Fowler Equations, International Journal of Information and Systems Sciences, Vol.3,No.2,pp.243-250(2007).

5.Ouda E. H. and Ahmed I.N., Numerical Methods for Solving Optimal Control Problem Using Scaling Boubaker Function, Al-Qadisiyah Journal of Pure Science,Vol.25,Issue(2),pp.7889(2020).

6.Keyanpour M. and Azizsefat M., Numerical Solution of Optimal Control Problems by An Iterative Scheme, Advanced Modeling and Optimization, Vol. 13(1), (2011).

7.Ramezanpour H. et al., An Iterative Procedure for Optimal Control of Bilinear Systems, International Journal of Instrumentation and Control Systems (IJICS),Vol. 2, No.1,(2012).

8.Elaydi H. et al., An Iterative Technique for Solving Nonlinear Optimal Control Problems Using Legendre Scaling Function, International Journal of Emerging Technology and Advanced Engineering, Vol.2,Issue (6), (2012).

9.Jaddu H. and Majdalawi A., An Iterative Technique for Solving a Class of Nonlinear Quadratic Optimal Control Problems Using Chebyshev Polynomials, International Journal of Intelligent Systems and Applications, Vol.6,pp.53-57(2014).

10.Jaddu H. and Majdalawi A., Recursive Approximation Method for Solving Constrained Nonlinear Optimal Control Problems Using Legendre Polynomials, International Journal of Soft Computing and Engineering Vol.5,Issue(2),pp.41-47(2015). 
11.Eskandari M. J. et al., Solving the Non-linear Quadratic Optimal Control Problem in Accordance with the Standard Variational Calculus Method (VCM) and Based on Variational Iteration Method(VIM), International Journal of Review in Life Sciences,Vol.5(8),pp.1678-1686(2015).

12.Ramezani M., Numerical Solution of Optimal Control Problems by Using a New Second Kind Chebyshev 's Wavelet, Computational Methods for Differential Equations, Vol.4,No.2,pp.162169(2016).

13.Shihab S. N. and Delphi M. M., State Parameterization Basic Spline Functions for Trajectory Optimization, Journal of Nature, Life and Applied Sciences,Vol.3,Issue(4),pp.110-119(2019).

14.Ibraheem S. F., Ouda E. H. and Ahmed I. N., Indirect Method for Optimal Control Problem Using Boubaker Polynomial, Baghdad Science Journal, Vol.13(1),pp.1-7, (2016).

15. Ouda E. H., A New Approach for Solving Optimal Control Problems Using Normalized Boubaker Polynomials, Emirates Journal for Engineering Research,Vol.23(4),pp.33-38(2018).

16.Vazquez-Leal H., Boubaker K., Hernandez L. and Huerta-Chua J., Approximation for Transient of Nonlinear Circuits Using RHPM and BPES Methods, Hindawi, Jour. Elect. Comp. Eng. Vol. (2013)(973813),pp.1-6.

17.Boubaker K.; On modified Boubaker polynomials: Some Differential and Analytical Properties of the New Polynomials, Journal of Trends in Applied Science Research,Vol.2,Issue(6),pp.540$544(2007)$.

18.Ahmed I. N. and Ouda E. H., Numerical Methods for Solving Optimal Control Problem Using Scaling Boubaker Function, Al-Qadisiyah Journal of Pure Science,Vol.25,Issue(2),pp.Math.7889(2020).

19.Delphi M. and Shihab S., Modified Iterative Algorithm for Solving Optimal Control Problems, Open Science Journal of Statistics and Applications, Vol.6(2),pp.20-27,(2019).

20.Mehne H. H. and Borzabadi A. H., A Numerical Method for Solving Optimal Control Problems Using State Parameterization, Numer . Algor., Vol.42, PP.165-169,(2006).

21.Kafash B., Delavarkhalafi S. M.; and Boubaker K.; A Numerical Approach for Solving Optimal Control Problems Using the Boubaker Polynomials Expansion Scheme, Journal of Interpolation and Approximation in Scientific Computing, pp.1-18,(2013). 